\title{
Measuring the collateral network pressure to minimize paraplegia risk in thoracoabdominal aneurysm resection
}

\author{
Christian D. Etz, MD, PhD, ${ }^{\text {a,b }}$ Stefano Zoli, MD, ${ }^{\mathrm{b}}$ Moritz S. Bischoff, MD, ${ }^{\mathrm{b}}$ Carol Bodian, DrPH, ${ }^{\mathrm{c}}$ \\ Gabriele Di Luozzo, MD, ${ }^{\mathrm{b}}$ and Randall B. Griepp, $\mathrm{MD}^{\mathrm{b}}$
}

\begin{abstract}
Objective: To minimize paraplegia during thoracoabdominal aortic aneurysm repair, the concept of the collateral network was developed. That is, spinal cord perfusion is provided by an interconnecting complex of vessels in the intraspinal, paraspinous, and epidural space and in the paravertebral muscles, including intercostal and lumbar segmental as well as subclavian and hypogastric arteries.
\end{abstract}

Methods: Collateral network pressure was measured with a catheter in the distal end of a ligated segmental artery in pigs and human beings.

Results: In the pig, collateral network pressure was $75 \%$ of the simultaneous mean aortic pressure. With complete segmental arterial ligation, it fell to $27 \%$ of baseline, recovering to $40 \%$ at 24 hours and $90 \%$ at 120 hours. Spinal cord injury occurred in approximately $50 \%$ of animals. When all segmental arteries were taken in 2 stages a week apart, collateral network pressure fell only to $50 \%$ to $70 \%$ of baseline, and spinal cord injury was rare. In human beings, baseline collateral network pressure also was $75 \%$ of mean aortic pressure, fell in proportion to the number of segmental arteries ligated, and began recovery within 24 hours. Collateral network pressure was lower with nonpulsatile distal bypass than with pulsatile perfusion.

Conclusions: After subtraction of a measure of spinal cord outflow pressure (cerebrospinal fluid pressure or central venous pressure), collateral network pressure provides a clinically useful estimate of spinal cord perfusion pressure. (J Thorac Cardiovasc Surg 2010;140:S125-30)

A logical initial step in the prevention of spinal cord injury (SCI) resulting from repair of thoracoabdominal aneurysms was the effort to identify segmental arteries (SAs) critical for spinal cord perfusion during surgery by means of somatosensory evoked potential (SSEP) and motor evoked potential (MEP) monitoring, both in the laboratory and in clinical practice. In our experience, however, sacrifice of all the segmental vessels (intercostal and lumbar) in experimental animals caused SCI in only half of the pigs studied. ${ }^{1}$ Clinically, extensive SA sacrifice resulted in rates of neurologic injury as low as or lower than those seen in contemporary series of aortic resections of comparable extent in which SAs were routinely reimplanted. ${ }^{2,3}$ Thus both in human beings and in pigs, extensive SA sacrifice without any SA reattachment resulted in normal neurologic function in most cases.

An extensive interconnected collateral network would explain the resiliency of the spinal cord circulation, but the characteristics of this network-and even its existence-

\footnotetext{
From the Department of Cardiac Surgery, ${ }^{a}$ Leipzig Heart Center, University of Leipzig, Leipzig, Germany; and Departments of Cardiothoracic Surgery, ${ }^{\mathrm{b}}$ and Anesthesiology, ${ }^{\mathrm{c}}$ Mount Sinai School of Medicine, New York, NY.

Supported by grant HL045636 from the National Heart, Blood and Lung Institute.

Disclosures: Christian D. Etz, Stefano Zoli, Moritz Bischoff, Carol Bodian, Gabriele

Di Luozzo, and Randall B. Griepp have nothing to disclose with regard to commercial support.

Received for publication April 27, 2010; accepted for publication July 12, 2010.

Address for reprints: Christian D. Etz, MD, PhD, Mount Sinai School of Medicine, Department of Cardiothoracic Surgery, One Gustave L. Levy Place, PO Box 1028,

New York, NY 10029 (E-mail: christian.etz@mountsinai.org). $0022-5223 / \$ 36.00$

Copyright (c) 2010 Published by Elsevier Inc. on behalf of The American Association for Thoracic Surgery

doi:10.1016/j.jtcvs.2010.07.040
}

have not previously been thoroughly explored. The first clue to the presence of this collateral network was the observation that the extrasegmental input from the subclavian and hypogastric arteries is of great importance to maintaining spinal cord viability after extensive SA sacrifice. ${ }^{4}$ Acrylic injection studies have since further characterized a rich paraspinous and intraspinal circulation that undergoes a series of adaptations to compensate for loss of SA input. ${ }^{5,6}$

In the course of investigating spinal cord perfusion and preservation, a technique for measuring the pressure in the collateral network directly was developed in experimental animals and was subsequently adapted for use in human patients. ${ }^{1,7}$ This technique allows close observation of the serial physiologic changes that occur during recovery from extensive SA sacrifice and enables assessment of the effectiveness of the collateral circulation. It has also permitted studies of the time required for restoration of collateral network pressure (CNP) to normal baseline values, which has led to the idea that dividing SA sacrifice into 2 operations might allow routine complete SA sacrifice without neurologic injury. The experiments to evaluate this hypothesis are reported here. The technique for direct monitoring of CNP will likely prove valuable clinically, both during operation and thereafter, and will help to reduce further the risk of paraplegia after repair of extensive thoracoabdominal aneurysms.

\section{MATERIALS AND METHODS \\ Experimental Studies}

The studies were carried out in juvenile Yorkshire pigs weighing 20 to $30 \mathrm{~kg}$. The pigs were cared for in an approved facility meeting guidelines 

Abbreviations and Acronyms
$\mathrm{CNP}=$ collateral network pressure
$\mathrm{CPB}=$ cardiopulmonary bypass
$\mathrm{CSF}=$ cerebral spinal fluid
$\mathrm{CVP}=$ central venous pressure
MAP $=$ mean arterial pressure
MEP $=$ motor evoked potential
$\mathrm{SA}=$ segmental artery
$\mathrm{SCI}=$ spinal cord injury
$\mathrm{SCPP}=$ spinal cord perfusion pressure
SSEP $=$ somatosensory evoked potential

set forth by the National Institutes of Health, the Guide for the Care and Use of Laboratory Animals (www.nap.edu/catalog/5140.html). All protocols were approved by the appropriate institutional review board. The anesthetic and surgical protocols have been described in detail in previous publications. $^{1,8}$

Serial SA sacrifice was carried out at $32^{\circ} \mathrm{C}$. A catheter was inserted into the distal stump of the SA at the 1st lumbar segmental artery and tunneled under the skin of the back. This catheter was used to measure CNP-which we consider to be closely related to spinal cord perfusion pressure (SCPP) both intraoperatively and postoperatively. The catheter was flushed with heparin every 12 hours to ensure patency. An aortic catheter, similarly maintained, allowed monitoring of mean arterial pressure (MAP); the CNP could therefore be expressed as a percentage of MAP.

CNP and arterial pressures were tracked for 5 days postoperatively. Functional recovery after SA sacrifice was assessed by means of a modified Tarlov score assigned after blinded inspection of daily videotaped recordings of each pig's postoperative behavior. The modified Tarlov scale has previously been described in detail: 9 indicates fully normal neurological function, whereas a score of 0 is given for complete paraplegia. ${ }^{8}$

After preliminary studies that identified the time course of the changes in CNP following after SA ligation, 2 protocols, each involving 20 pigs, were carried out. In the first, SA sacrifice from T4 to T13 was followed after 1 week by sacrifice of all lumbar SAs, and results were compared with contemporaneously randomized control pigs in which all thoracic and lumbar SAs were ligated in a single operation. In the second protocol, the 2-stage operation began with sacrifice of all lumbar SAs, followed by thoracic SA ligation 1 week later, also with randomized contemporaneous control pigs in which all the SAs were sacrificed in a single procedure. ${ }^{9}$

\section{Clinical Observations}

The clinical observations were obtained from 13 patients who underwent descending thoracic or thoracoabdominal aneurysm resection. The details of these patients' surgical procedures, measures for spinal cord protection, and anesthetic management have previously been published. ${ }^{10}$ The surgery was carried out with either moderate hypothermia $\left(32^{\circ} \mathrm{C}\right)$ with distal partial cardiopulmonary bypass $(\mathrm{CPB})$ or hypothermic circulatory arrest at an esophageal temperature of $12^{\circ} \mathrm{C}$ to $15^{\circ} \mathrm{C}$. A mean of $10 \pm 3$ SAs were ligated.

For assessment of CNP, a catheter was inserted into the stump of an intercostal or lumbar vessel in the center of the planned resection. The remaining SAs in the anticipated resected segmented were serially clipped and ligated. Cerebral spinal fluid (CSF) drainage and MEP and SSEP monitoring were used as previously described. ${ }^{3}$ The CNP monitoring was carried out in several patients for 24 hours postoperatively and in 1 patient for as long as 72 hours. No complications from CNP catheter placement or withdrawal were encountered.

\section{RESULTS \\ Experimental Studies}

When all SAs were taken concurrently, $40 \%$ to $60 \%$ of pigs had a full functional recovery, whereas the remainder showed some evidence of SCI. The pressure response of the collateral network to the sacrifice of SA input is seen in Figure 1. The CNP, which began at 60 to $70 \mathrm{~mm} \mathrm{Hg}$ at the start of SA sacrifice, fell to 20 to $25 \mathrm{~mm} \mathrm{Hg}$ after all the SAs had been ligated and reached a nadir at 5 postoperative hours. CNP then gradually began to recover by 24 hours, returning to baseline by 120 hours. Although no difference in the pattern of CNP recordings between pigs that recovered and those that sustained SCI was seen in this experiment, the MAP at 5 hours was slightly but significantly higher in the pigs that recovered. ${ }^{1}$

Figure 2 shows the curve of CNP recovery in animals that underwent single-stage extensive SA ligation compared with the response of those with SA sacrifice in 2 stages, 1 week apart. The control graph-concurrent single-stage ligation of all SAs-in the 2-stage experiments was the same as the pattern in Figure 1. If thoracic SA sacrifice preceded lumbar SA ligation by 1 week, the decrease in CNP after the second stage was much less marked than if all SAs were sacrificed in a single procedure, remaining above $40 \mathrm{~mm} \mathrm{Hg}$ (Figure 2). If lumbar SA ligation was carried out as the first stage, it was possible to measure CNP (in the 1st lumbar segmental artery) during and after the first stage as well as to monitor the second stage of the 2-stage procedure. After the first stage of the lumbar-first procedure, the decrease in CNP was approximately $45 \%$ of the drop in CNP in the single-stage SA ligation group; after the second stage, as in the thoracic-first protocol, the decrease in CNP was much less marked.

The CNP patterns in the pigs after single-stage and 2stage protocols are reflected by the results of behavioral evaluation seen in Figure 3. It is apparent that all the pigs recovered quickly - and most of them completely—after all the SAs were ligated in both of the 2-stage protocols. After the single-stage (control) procedure, however, recovery was generally slower and less complete, and several pigs in each experiment suffered complete paraplegia.

\section{Clinical Observations}

Among the patients who had catheter placement during resection of an extensive descending thoracic or thoracoabdominal aneurysm, the relationship between MAP and CNP was not as constant from one individual to the next as it was in the experimental animals. According to intraoperative measurements, the gradient between MAP and CNP was higher in the hypertensive patients. Thus, in hypertensive patients, a higher MAP was required to maintain CNP values considered to be consistent with adequate spinal cord perfusion. 


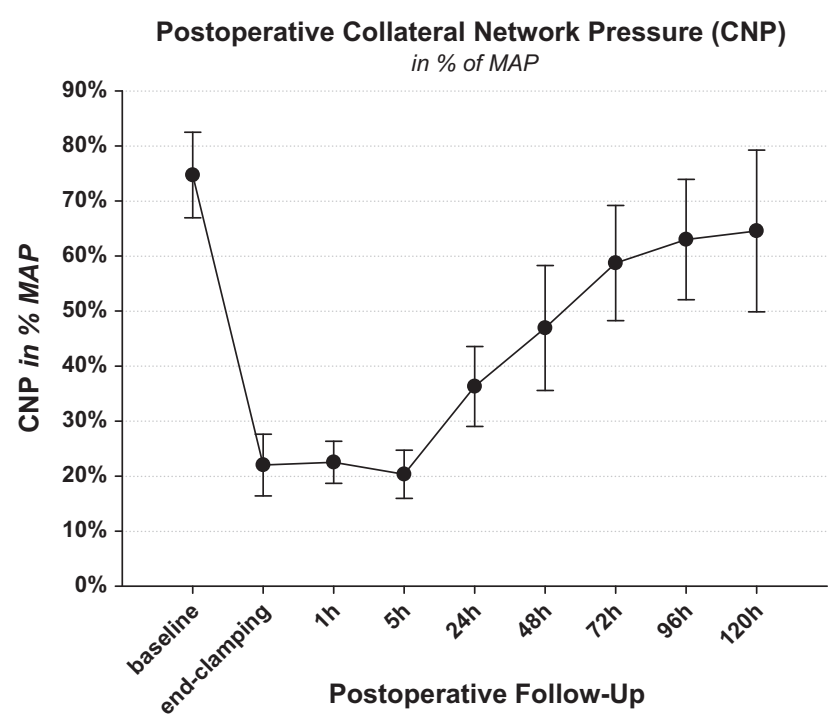

FIGURE 1. Collateral network pressure $(C N P)$ measured in distal end of 1 st lumbar segmental artery in 10 pigs after ligation of all segmental vessels. $M A P$, Mean arterial pressure.

During surgery, the CNP dropped to about $60 \%$ of MAP with SA sacrifice and then dropped further during CPB. Once pulsatile perfusion was restored, CNP began to return to pre-CPB values (Figure 4).
Postoperative CNP monitoring in patients suggests that SCPPs remained low for several hours after extensive SA ligation - as they did in the pigs-before gradually beginning to climb at about 24 hours (Figure 5). Because there is evidence that a high central venous pressure (CVP) or CSF pressure will impair spinal cord perfusion, SCPP may best be estimated by subtracting CVP or CSF pressure from CNP. When the data were thus evaluated, a clear separation of uninjured patients from the single patient who suffered paraparesis emerged, with significantly different CNP curves (Figure 5).

In defining an adequate minimum SCPP, one can use as a guide for a safe number the minimum value calculated for SCPP intraoperatively at a time when the patient's MEPs are robust and neurologic function is thus known to be intact. In the patient in Figure 5 in whom paraparesis occurred, the SCPP was recognized to be low but could not be raised, principally because morbid obesity resulted in a high CVP and corresponding high CSF pressure that did not respond to measures aimed at their reduction.

\section{DISCUSSION}

The results of both experimental and clinical measurements of the pressure within the collateral network indicate that CNP falls to low levels once all the SAs have been

Collateral Network Pressure (CNP, L1)

after extensive single vs. two stage SA sacrifice
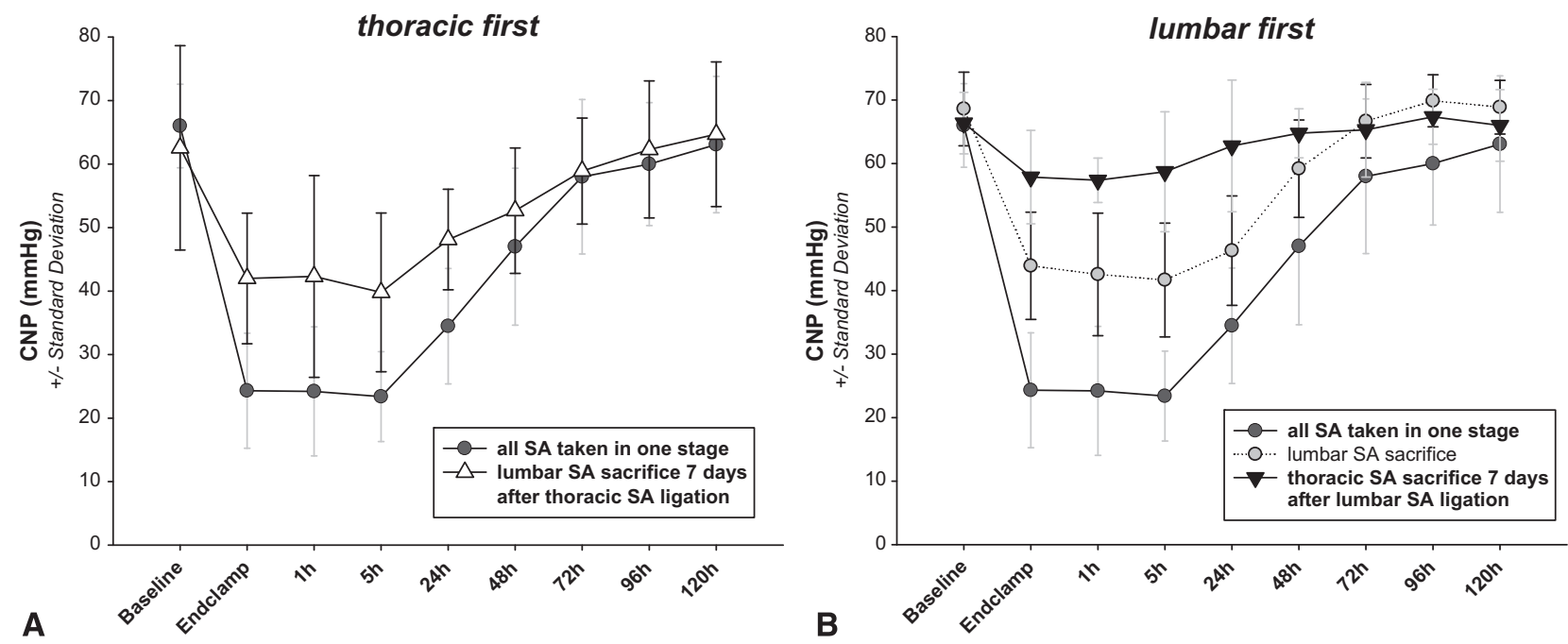

FIGURE 2. Collateral network pressure (CNP) tracings at 1st lumbar segmental artery, as in Figure 1. A, In thoracic-first protocol, pressure is shown for 10 pigs after lumbar segmental arterial ligation. Thoracic segmental arteries ( $S A s)$ had been sacrificed 7 days previously. Shown for comparison is collateral network pressure curve in 10 randomly intercalated control pigs that underwent thoracic and lumbar segmental arterial ligation as single procedure. Drop in collateral network pressure in experimental animals when lumbar segmental arteries were ligated at second procedure was half magnitude of drop in collateral network pressure when lumbar and thoracic segmental arteries were ligated in single stage. B, In lumbar-first protocol, collateral network pressure was measured at 1st lumbar segmental artery in 10 pigs after lumbar segmental arterial ligation, and in same pigs 7 days later, after thoracic segmental arterial sacrifice. Shown for comparison is collateral network pressure curve from 1st lumbar segmental artery in 10 randomly intercalated pigs that underwent ligation of all segmental arteries in singles stage. Drop in collateral network pressure when only lumbar segmental arteries are ligated in first stage is half drop when all segmental arteries are sacrificed at once. When thoracic segmental arteries are ligated 1 week later, drop in collateral network pressure is 1 quarter drop in control, single-stage group. 
Two staged thoracic first
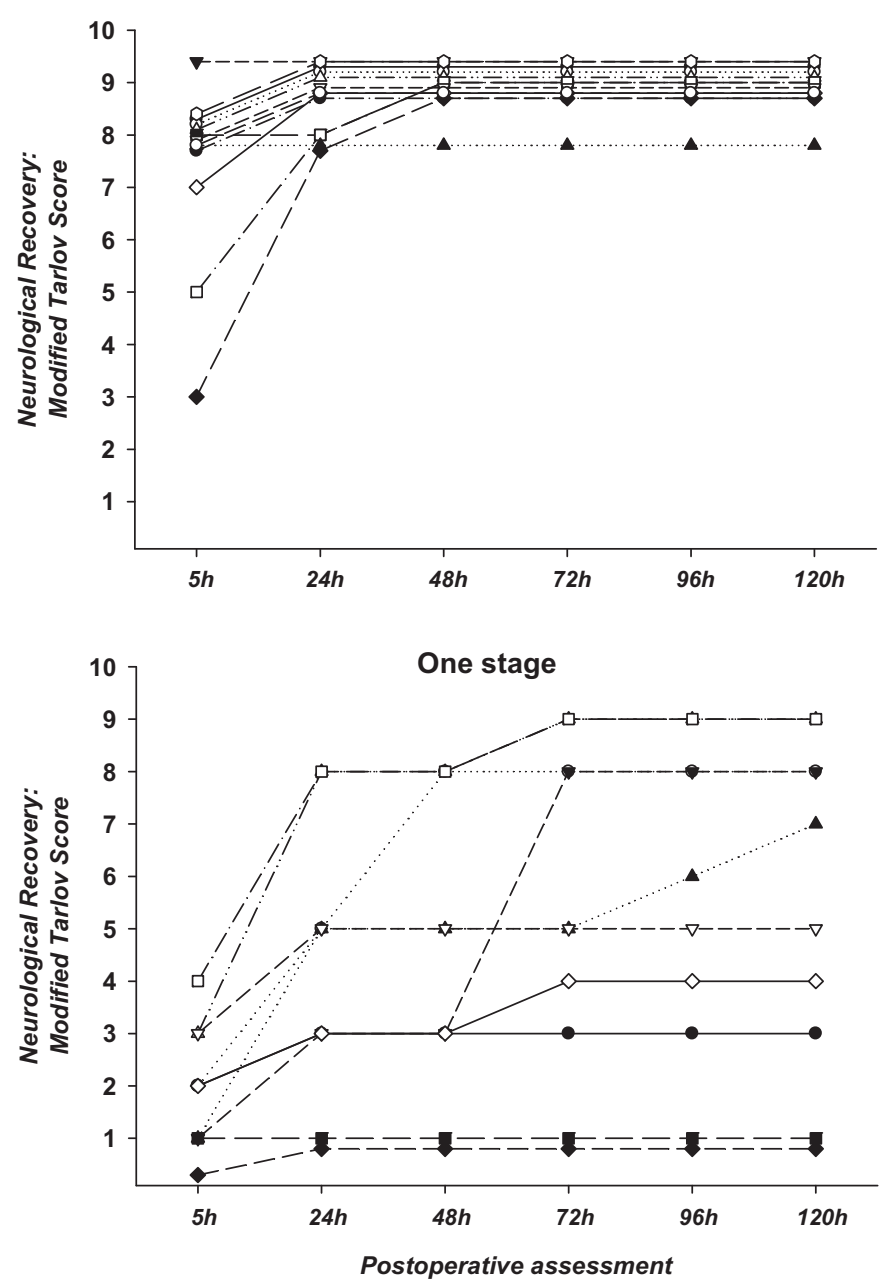

Two staged lumbar first
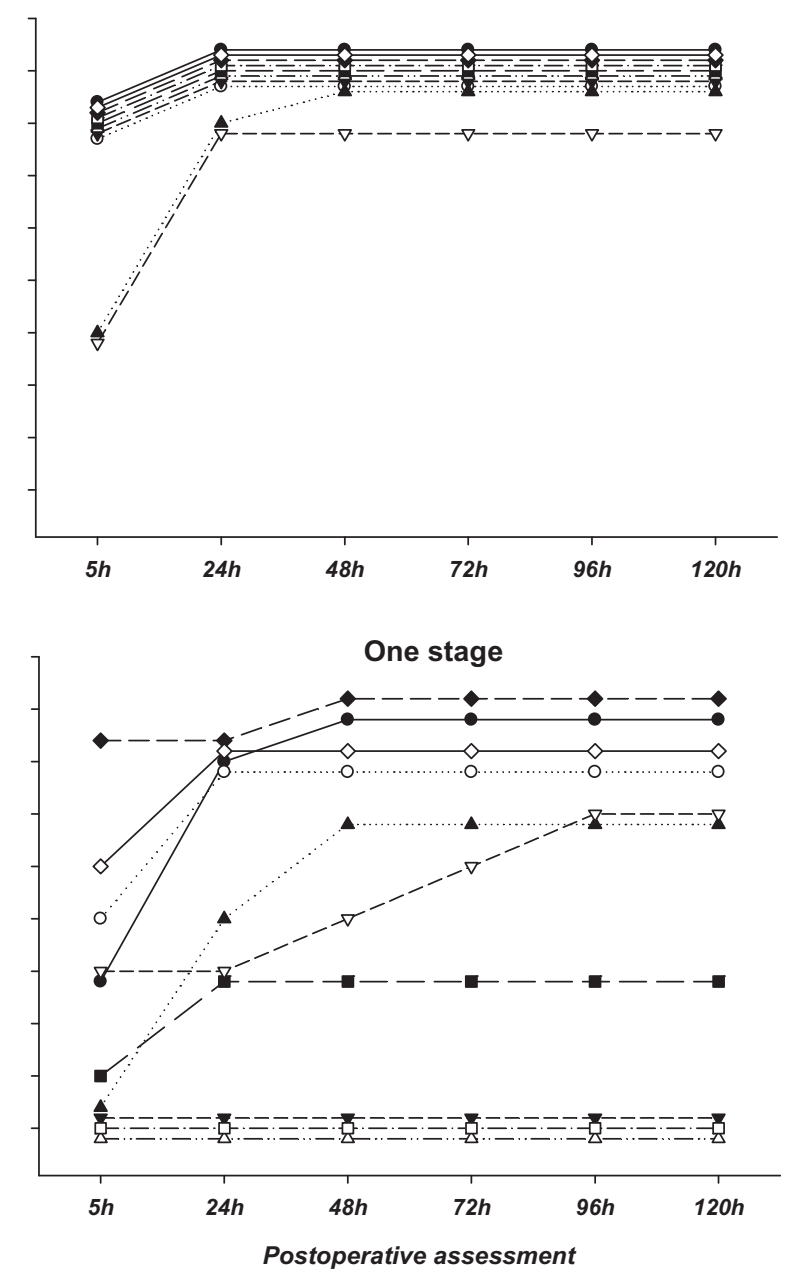

FIGURE 3. Modified postoperative Tarlov score from 5 hours to 5 days. Score of 9 is completely normal, and pigs with score of 1 had no hind limb movement whatsoever. Score of 4 indicates ability to get up and stand. Only 1 pig in each 2 -stage group had less than total recovery (upper 2 graphs). In 2 control groups with single-stage procedures, only 9 of 20 animals could ambulate satisfactorily (lower 2 graphs).

ligated but recovers beginning at 24 hours, regardless of functional outcome. In pigs, the lowest pressures appear to occur about 5 hours after SA ligation, coinciding with rewarming from mild hypothermia and with movement during awakening from anesthesia, both of which increase metabolic demand. In patients, a low CNP often persists for nearly 24 hours after extensive SA sacrifice, although the pattern varies, and a nadir at 5 hours is not always observed.

Clearly more data are necessary to define the spectrum of recovery of CNP in patients. Nevertheless, it is encouraging that in a very small group of patients, separation between injured and intact outcomes is possible by estimating SCPP as the CNP minus the CVP or CSF pressure. It remains to be ascertained whether there is a threshold SCPP common to most patients or whether a unique safe minimum SCPP must be estimated in each individual by ascertaining during surgery the CNP at which MEPs are present and robust.
Experimentally, if SA ligation is carried out in 2 stages, allowing several days of recovery and stabilization before the second stage, then the drop in pressure when the last of the SAs is taken is much less marked, and functional recovery is significantly better. The lower incidence of SCI with extensive thoracoabdominal aneurysm repair when carried out in 2 stages rather than during a single operation has also been suggested by several clinical studies. ${ }^{10,11}$

SCPP seems to recover even in patients with SCI, but the magnitude of its fall and the duration of low values seem to correlate with the occurrence and severity of SCI. In clinical practice, it appears that a higher MAP is required to produce an adequate SCPP in patients who have been hypertensive before surgery. The possibility of concurrent intraoperative MEP and postoperative SSEP monitoring will refine our ability to be sure when perfusion is adequate, and experience and study will further validate the utility of measuring SCPP. 


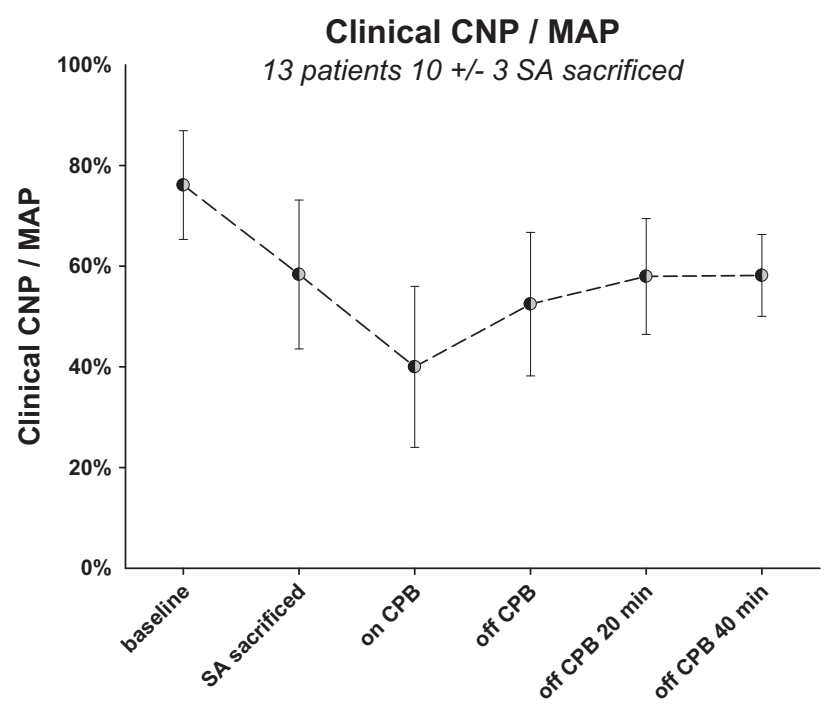

FIGURE 4. Collateral network pressure (CNP) expressed as percentage of mean arterial pressure $(M A P)$ in 13 patients undergoing extensive descending thoracic or thoracoabdominal aortic resections. Collateral network pressure catheter was placed as close as possible in segmental artery (SA) in middle of resected aortic segment. Significant pressure drop was seen with segmental arterial ligation, followed by further drop with cardiopulmonary bypass $(C P B)$. This is average measured pressure during partial cardiopulmonary bypass providing distal perfusion in patients in whom this modality was used, and pressure on complete cardiopulmonary bypass during cooling and rewarming in patients in whom complete hypothermic circulatory arrest was used. With discontinuation of cardiopulmonary bypass and restoration of pulsatile perfusion, collateral network pressure values increased to post-segmental arterial ligation levels. Somatosensory and motor evoked potentials were present in all patients at all times except during hypothermic circulatory arrest and initial warming after hypothermic circulatory arrest.

Because a high CVP or CSF pressure also impairs spinal cord perfusion, the calculated SCPP (which incorporates these factors as well as CNP) seems the best tool for clinical monitoring of the adequacy of spinal cord perfusion. The fact that CNP and calculated SCPP can be tracked with hemodynamic monitoring techniques familiar to all operating room personnel as well as to the Intensive care unit nurses caring for the patients postoperatively makes it potentially a much more universally applicable technique than SSEP or MEP monitoring, both of which require a skilled neurophysiologist. We think it is likely that measurement of CNP and calculation of SCPP will prove useful in guiding both intraoperative and postoperative management.

The fact that the SCPP begins to recover significantly within 24 hours gives even relatively labor-intensive strategies to determine the minimum safe levels and then to work to maintain these levels during this relatively short vulnerable interval appeal as a worthwhile endeavor. Among the strategies whose effectiveness can likely be monitored with the SCPP are choice of pressors, regulation of fluid balance, optimization of CSF drainage, manipulation of body

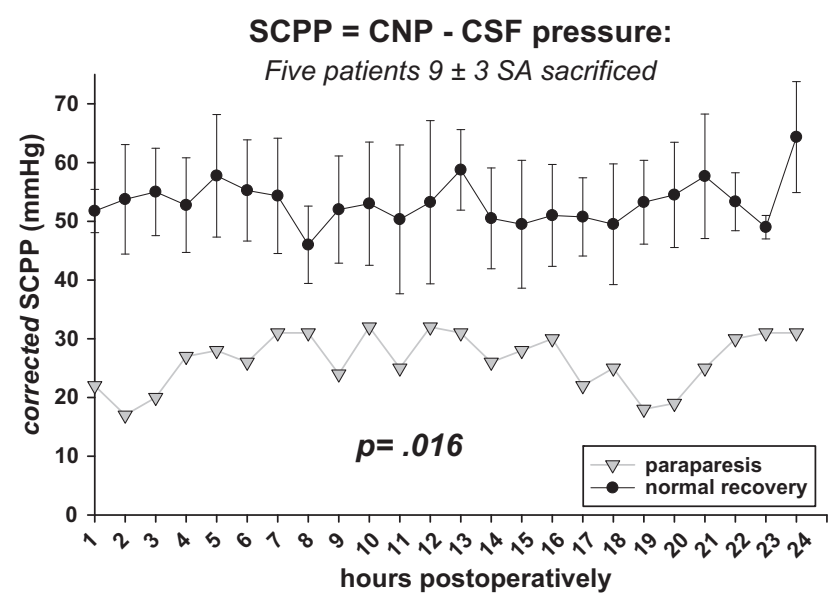

FIGURE 5. Spinal cord perfusion pressure ( $S C P P$ ), as explained in text, during first 24 postoperative hours in 5 patients. Four patients who sustained no spinal cord injury are contrasted with single patient who had paraparesis. In injured patient, spinal cord perfusion pressure reflected slightly low collateral network pressure $(C N P)$ and very high central venous pressure $(C V P)$ and cerebrospinal fluid $(C S F)$ pressure, probably secondary to morbid obesity necessitating high ventilatory pressures.

temperature, and inhibition of shivering and other muscular movement that may provoke steal from the spinal cord circulation.

Finally, it should be possible to measure CNP in patients undergoing stent grafting for aortic disease. It should not be overwhelmingly difficult to position a right-angle catheter in an appropriate SA just before deployment of the stent-graft. CNP could be monitored and the catheter withdrawn after 48 to 72 hours.

\section{CONCLUSIONS}

Clinically, CNP can be safely measured intraoperatively and for as long as 72 hours after surgery, and this technique could be implemented in conjunction with thoracic endovascular aortic repair. The intraoperative fall in CNP appears to be proportional to the number of SAs sacrificed. At the same MAP, CNP is lower during complete or partial CPB than with pulsatile perfusion off bypass, with recovery to baseline $\mathrm{CNP}$ in some patients as early as 24 to 48 hours. An estimate of SCPP (CNP minus CSF pressure or CVP) is likely to be useful in postoperative hemodynamic management and in predicting the likelihood of SCI. Both experimental studies and a retrospective analysis of patients who have undergone extensive SA sacrifice suggest that resection in 2 stages carries a much lower risk of SCI than does a single-stage operation of the same extent.

\section{References}

1. Etz CD, Homann TM, Plestis KA, Zhang N, Luehr M, Weisz DJ, et al. Spinal cord perfusion after extensive segmental artery sacrifice: can paraplegia be prevented? Eur J Cardiothorac Surg. 2007;31:643-8.

2. Griepp RB, Ergin MA, Galla JD, Lansman S, Khan N, Quintana C, et al. Looking for the artery of Adamkiewicz: a quest to minimize paraplegia after operations for 
aneurysms of the descending thoracic and thoracoabdominal aorta. $J$ Thorac Cardiovasc Surg. 1996;112:1202-15.

3. Etz CD, Halstead JC, Spielvogel D, Shahani R, Lazala R, Homann TM, et al. Thoracic and thoracoabdominal aneurysm repair: is reimplantation of spinal cord arteries a waste of time? Ann Thorac Surg. 2006;82: 1670-7.

4. Strauch JT, Spielvogel D, Lauten A, Zhang N, Shiang H, Weisz D, et al. Importance of extrasegmental vessels for spinal cord blood supply in a chronic porcine model. Eur J Cardiothorac Surg. 2003;24:817-24.

5. Etz CD, Kari FA, Mueller CS, et al. The collateral network concept: a reassessment of the anatomy of spinal cord perfusion. J Thorac Cardiovasc Surg. In press.

6. Etz CD, Kari FA, Mueller CS, Brenner R, Lin HM, Griepp RB. Remodeling of the arterial collateral network after experimental segmental artery sacrifice. $J$ Thorac Cardiovasc Surg. In press.
7. Etz CD, Di Luozzo G, Zoli S, Lazala R, Plestis KA, Bodian CA, et al. Direct spinal cord perfusion pressure monitoring in extensive distal aortic aneurysm repair. Ann Thorac Surg. 2009;87:1764-74.

8. Etz CD, Homann TM, Luehr M, Kari FA, Weisz DJ, Kleinman G, et al. Spinal cord blood flow and ischemic injury after experimental sacrifice of thoracic and abdominal segmental arteries. Eur J Cardiothorac Surg. 2008;33:1030-8.

9. Zoli S, Etz CD, Roder F, Brenner RM, Bodian CA, Kleinman G, et al. Experimental two- stage simulated repair of extensive thoracoabdominal aneurysms reduces paraplegia risk. Ann Thorac Surg. 2010;90:722-9.

10. Etz CD, Zoli S, Mueller CS, Bodian CA, Di Luozzo G, Lazala R, et al. Staged repair significantly reduces paraplegia rate after extensive thoracoabdominal aortic aneurysm repair. J Thorac Cardiovasc Surg. 2010;139:1464-72.

11. Coselli JS, Poli de Figueiredo LF, LeMaire SA. Impact of previous thoracic aneurysm repair on thoracoabdominal aortic aneurysm management. Ann Thorac Surg. 1997;64:639-50. 